\title{
Homochirality in space - Selective enrichment of chiral molecules on chiral surfaces
}

\author{
G. Marloie ${ }^{1}$, F. Pauzat ${ }^{1}$, Y. Ellinger ${ }^{1}$, A. Markovits ${ }^{1}$ and J. Pilmé ${ }^{1}$ \\ ${ }^{1}$ Laboratoire de Chimie Théorique, UPMC (Univ. Paris VI), UMR-CNRS 7616, France
}

\begin{abstract}
Life on Earth, as we know it to day, is inseparable from homochirality. The presence of an enantiomeric excess in carbonaceous chondrites is well documented; among the different hypotheses considered to explain this undisputed fact, we chose to investigate the possibilities open by a selective adsorption on a chiral surface that would engage a process of enantiomeric enrichment. The chiral surface is that of the $\alpha$-quartz $\left\{\begin{array}{ll}10 & \overline{1} 0\end{array}\right\}$ and the chiral molécule is lactic acid, $\mathrm{HOCH}\left(\mathrm{CH}_{3}\right) \mathrm{COOH}$. In this theoretical work we rely on numerical simulations based on Density Functional Theory (DFT) that proved efficient in the super-molecule and periodic approaches to this category of phenomena. In view of the different types of adsorption sites, a statistical treatment was necessary; it shows that (S)-lactic acid is preferentially adsorbed with a selectivity of $\sim 0.7 \mathrm{kcal} / \mathrm{mol}$ with respect to the $(\mathrm{R})$ isomer.
\end{abstract}

\section{Introduction}

The presence of an enantiomeric excess in the organic matter of well defined families of meteorites [1-3] is a robust fact. However, not only is the origin of this enantiomeric excess unknown but the emergence itself of the first chiral molecule is still an unanswered question.

Two enantiomers have identical internal energies. When adsorbed on a same chiral surface, the complexes obtained are no longer enantiomers, but diastereoisomers. These diastereoisomers do not have the same energy. Assuming that we have a chiral interstellar surface at hand, we can consider the possibility to adsorb selectively one of the two enantiomers and thus engage a process of enantiomeric enhancement. Here we address the question of how much the adsorption energy differs from one enantiomer to the other. To this end we rely on numerical simulations based on the methods of quantum chemistry, namely Density Functional Theory (DFT) that proved efficient in the super-molecule (cluster) and periodic approaches to this category of phenomena [4].

\section{Principle and modeling}

In the cluster model of the chiral $\alpha$-quartz, the $\left\{\begin{array}{ll}10 & \overline{1} 0\end{array}\right\}$ active surface is obtained by an appropriate cut of the crystal structure. To account for the nature of the interstellar grains, the active surface is fully hydroxylated. The dangling bonds of the non active surfaces are saturated by hydrogen atoms. The positions of the hydrogen atoms are optimized to obtain the most stable structure.

In the periodic model of the chiral $\alpha$-quartz, the $\left\{\begin{array}{ll}10 & \overline{1} 0\end{array}\right\}$ active surface is directly obtained by the same cut of the crystal structure. The thickness of the infinite slab is equal to that of the model 
cluster with the dangling bonds at the bottom also saturated by hydrogen atoms. As in the cluster model, the active surface is fully hydroxylated and the positions of the hydrogen atoms optimized. To avoid spurious lateral interactions between adsorbates arising from in-plane replication, the surface of the unit cell was taken equal to $(9 \times 10) \AA^{2}$; to avoid interaction by translation in the vertical dimension, a distance of $25 \AA$ was imposed between two successive slabs.

\section{Statistical approach of the surface selectivity}

Exploratory studies of the adsorption of simple molecules have shown different adsorption energies for different adsorption points on the surface. It is therefore logical to consider that the complexity of the $\alpha$-quartz surface could potentially generate different adsorption sites with opposite selectivity for chiral molecules. A rapid exploration of intuitive sites confirmed that such possibility does exist. In this condition, a local adsorption is not representative of the real selectivity of a surface and may lead to erroneous conclusions. We propose that the selectivity of the surface, that appears to be a global property, be determined by a statistical approach. It is based on the following steps, illustrated here by the case of lactic acid:

A first screening is performed starting from a dense mesh of initial guesses of adsorption points (molecule + interacting atoms on the surface). Several hundreds of adsorption points are considered (1680 sites) and the structure of each of them is optimized using the semiempirical DFTB+ method (Tight Bonding approximation). The adsorption points are then regrouped by geometrical similarity, defining adsorption sites (15 groups with 6 major).

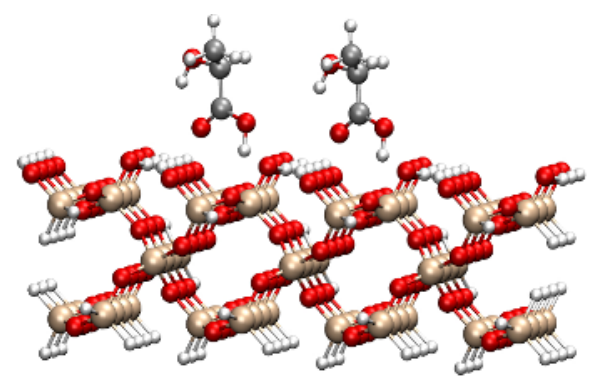

Example of 2 optimized positions grouped in the same adsorption site.

The geometry of the most populated sites (total $\geq 70 \%$ ) is re-optimized using the cluster model. The geometries thus obtained are then transferred to the periodic model to obtain the final adsorption energies. Different adsorptions energies are found according to the topology of the adsorption sites, showing the possibility of an opposite selectivity. The adsorption energy that is characteristic of the couple adsorbate/surface is obtained as the sum of the differences of the $\mathrm{R}$ and $\mathrm{S}$ adsorption energies of the most significant sites weighted by their occurrence numbers normalized to $100 \%$. On the $\left\{\begin{array}{ll}10 & \overline{1} 0\end{array}\right\}$ active surface of the a-quartz, the statistical approach presented here shows that (S)-lactic acid is preferentially adsorbed. However, the difference between the adsorption energies of the $\mathrm{R}$ and $\mathrm{S}$ enantiomers is rather small $(\sim 0.7 \mathrm{kcal} / \mathrm{mol})$. In the end, the selectivity, although real, does not seem large enough to induce a large enantiomeric excess of (S)-lactic acid in interstellar grains [5].

\section{References}

1. J.R. Cronin, S. Pizzarello, Science, 275, 951 (1997)

2. S. Pizzarello, J.R. Cronin, Nature, 394, 236 (1998)

3. S. Pizzarello, J.R. Cronin, Geochim. Cosmochim. Acta, 64, 329 (2000)

4. M. Lattelais, M. Bertin, H. Mokrane, et al., A\&A, 532, A12 (2011)

5. G. Marloie, F. Pauzat, Y. Ellinger (to be published) 